This item was submitted to Loughborough's Research Repository by the author.

Items in Figshare are protected by copyright, with all rights reserved, unless otherwise indicated.

\title{
A novel adaptive shading correction algorithm for camera systems
}

PLEASE CITE THE PUBLISHED VERSION

http://dx.doi.org/10.2352/ISSN.2470-1173.2016.18.DPMI-249

\section{PUBLISHER}

(C) Society for Imaging Science and Technology

\section{VERSION}

VoR (Version of Record)

\section{PUBLISHER STATEMENT}

This work is made available according to the conditions of the Creative Commons Attribution-NonCommercialNoDerivatives 4.0 International (CC BY-NC-ND 4.0) licence. Full details of this licence are available at: https://creativecommons.org/licenses/by-nc-nd/4.0/

\section{LICENCE}

CC BY-NC-ND 4.0

\section{REPOSITORY RECORD}

De Silva, Varuna, Viacheslav Chesnokov, and Daniel Larkin. 2019. "A Novel Adaptive Shading Correction Algorithm for Camera Systems”. figshare. https://hdl.handle.net/2134/24089. 


\section{A Novel Adaptive Shading Correction Algorithm for Camera Systems}

Varuna De Silva, Viacheslav Chesnokov, Daniel Larkin; Apical Ltd; Loughborough, United Kingdom

\begin{abstract}
Lens Vignetting is a common distortion in imaging systems where the intensity of the image decreases gradually away from the image center. Incorrect compensation of this luminance degradation often results in a more disturbing artifact called color shading, where the resulting image is left with shades of different colors. This paper presents a novel adaptive technique to compensate for lens Vignetting, while preserving color saturation and eliminating the need for per-unit calibration. As a consequence, the performance of automatic white balance algorithms have also been improved. The proposed algorithm is lightweight and realizable on FPGA architecture for real time Vignetting correction. The experimental results indicate a significant improvement in the quality of the corrected images. It is expected that the proposed algorithm will be implemented on mobile Image Signal Processors (ISPs) in the near future.
\end{abstract}

\section{Introduction}

Vignetting is the phenomenon that the brightness of an image gradually decrease radially away from the center of an image [1]. Lens Vignetting or lens shading is mainly caused due to the fact that the lens assembly will hinder the amount of light falling towards the border pixels of the CMOS sensor. If the radial intensity falloff can be accurately identified, the vignetting effect can be successfully compensated [2]. However, each camera unit (Lens + Sensor) that is manufactured is a unique product due to the manufacturing variations at sensor level in Color Filter Array (CFA) and in Micro-Lens, variation of IR cutoff filters and the cutoff frequency changes over the angle of incident light. Furthermore, assembly issues such as misalignment between the lens and sensor, or tilting of the lens will also contribute to per-unit variations of different sensors [3]. Therefore, due to such per-unit variations of camera units the vignetting correction function need to be tuned for each individual unit, which is infeasible especially for mass produced camera units such as those of mobile phones. Furthermore, vignetting function also changes with the light source by which a scene is illuminated [4].

If a single shading correction function is applied to all the camera units of a given type, without considering for per-unit variations, a residual effect know as color shading becomes visible in the resultant image. Color shading manifests as a color cast(s) on the image, which is quite undesirable. Therefore, an adaptive shading correction scheme is necessary to compensate for per-unit variations to correct lens shading. Figure 1 illustrates the minor variations in the vignetting function in two different sensors under three different light sources.

The traditional methods of correcting for shading often focus on correcting for a single sensor [5]. Vignetting function is either approximated by a polynominal or estimated in the lab environment by capturing flatfield images under different light sources. The vignetting function is then used to change the values of the pixels. However, such methods mainly focus on correcting for luminance shading and often neglect per-unit variations in camera units, which result in residual artifacts known as color shading[1][2]

While per-unit calibration on the other hand is quite an expensive process, state-of-the art shading correction techniques try to compensate for per-unit variations by adapting the vignetting function from one sensor to another. However, most of these methods rely on Auto White Balance (AWB) statistics to deduce the color temperature from the image, and then appropriately modify the vignetting function [3]. Another method known as Dynamic Mesh Shading Correction (DMSC) stores flatfields (mesh) captured under three different lighting sources, and then based on the color temperature the flatfields (mesh) at different temperatures are blended appropriately to find a suitable correcting function for the image.

Two problems can be identified with the current approaches. Certain techniques are non-adaptive, and thus oversee per-unit variations, and end up with color shading artifacts. While some of the more advanced adaptive techniques often rely on correct illuminant estimation, which often liable to fail.

To overcome the limitations of the current approaches, a new Adaptive Shading Correction (ASC) algorithm is proposed to remove the effects of lens shading. The objective of the approach is to adaptively correct each image for shading while considering the per-unit variations and the difference in light sources. Thus eliminating the need for per-unit calibration and the need for light source identification. The proposed ASC method is described in more details in the next section.

The rest of the paper is organized as follows; The next section describes the proposed algorithm, which followed by a section of results and discussions. Finally the paper is concluded in the conclusions section, with indications to future work.

\section{The Proposed Algorithm}

In this section the proposed algorithm is described in detail. The basic flow of the algorithm is also illustrated in Figure 2.

\section{Stage I: Generation of the Average Shading Mesh \\ Tertiary Heading}

In this stage, it is expected to capture flatfield shading images for different light sources, for several sensors of the same type. The resultant raw images are processed and averaged to generate a representative shading mesh. This will be known as average shading mesh, which is the basic vignetting function that will be adapted in stage IV.

\section{Stage II: Analysis of the RAW image}

After pre-processing the raw image (Bilinear Demosaicing, Grey World White Balance (GWWB), and down sampling) the algorithm will identify regions of similar color (hue) in the image. The image is segmented in to equal size blocks. A block is considered flat in hue, if there is low variation of hue in the. 


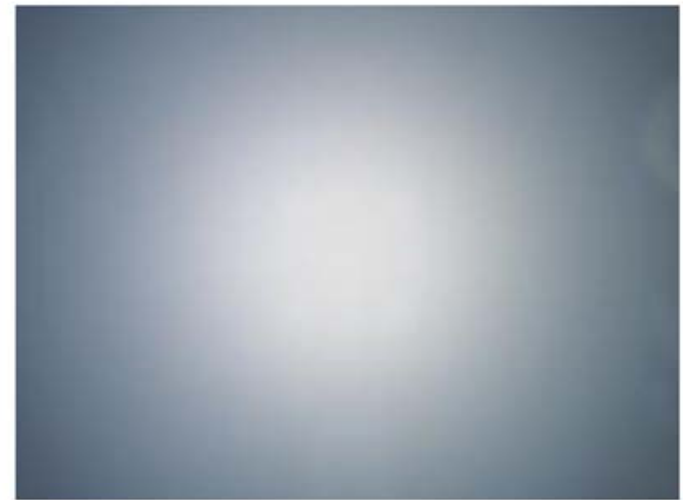

D30, Sensor 1

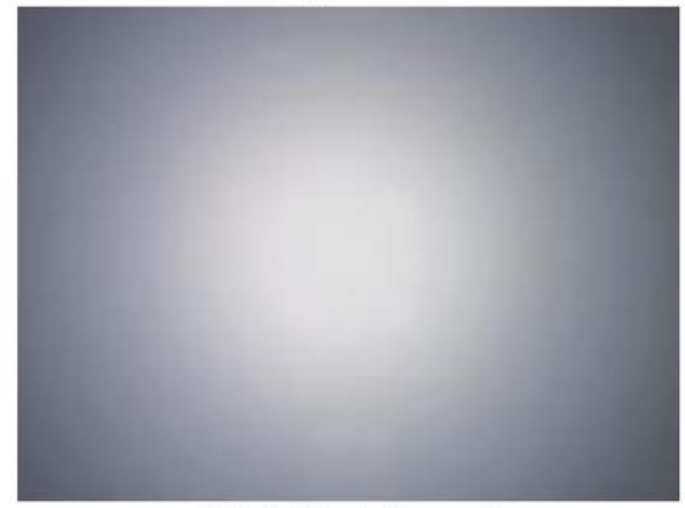

D65 (Philips), Sensor 1

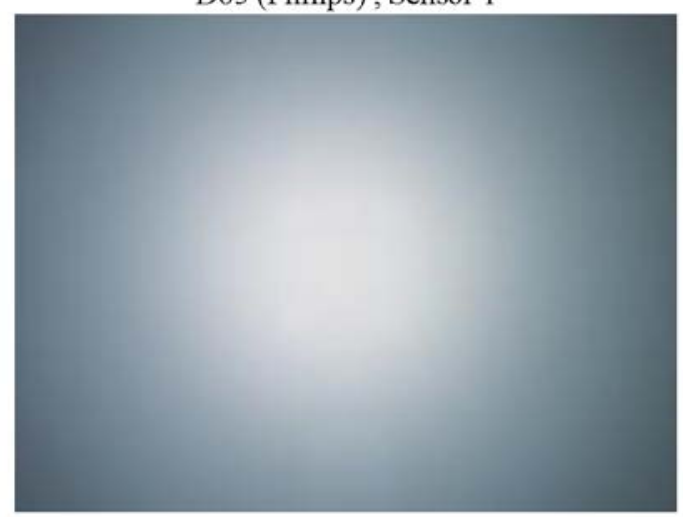

D65 (Macbeth), Sensor 1
D30, Sensor 5

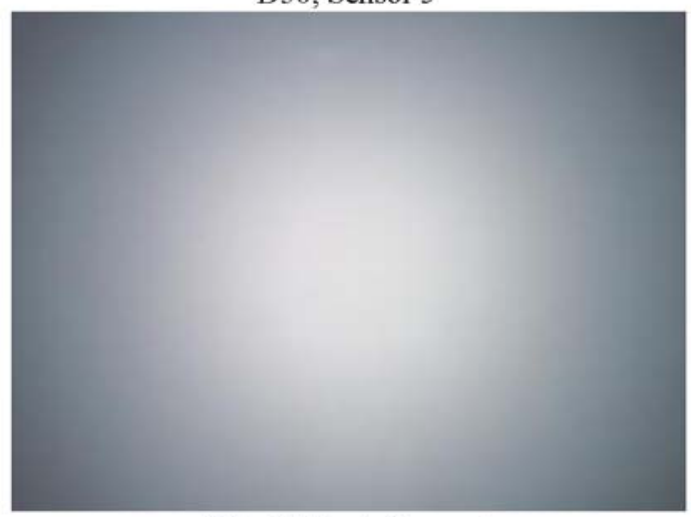

D65 (Philips), Sensor 5

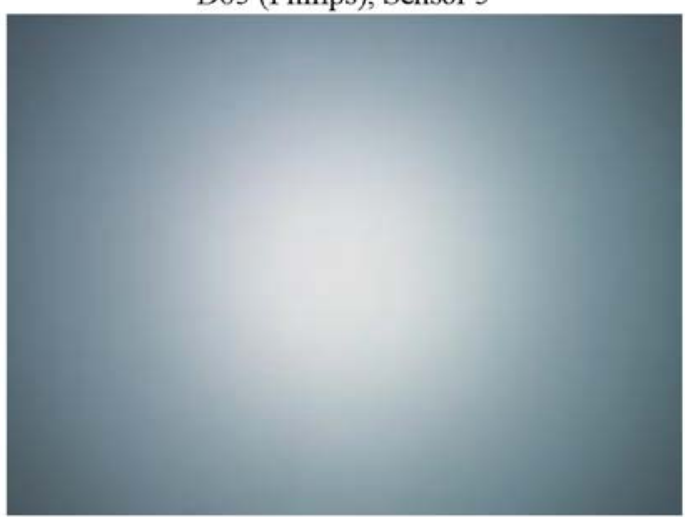

D65 (Macbeth), Sensor 5

Figure 1: The differences visible in a flatfield capture under different light sources

particular block. Variation of hue is measured by the hue variance, as shown in (1);

$\left.H_{r / g}=\frac{\sum_{\forall \text { pixels }}(r / g-\bar{r} / g}{g}\right)^{2}$

where $\mathrm{N}$ is the number of pixels in the block, $\mathrm{r}$ and $\mathrm{g}$ are the value of the red and green channels. Similarly variation of hue is measured for $\mathrm{b} / \mathrm{g}$ as well. A particular block is considered flat if the hue variation in both $\mathrm{r} / \mathrm{g}$ and $\mathrm{b} / \mathrm{g}$ is less than a particular threshold;

$F=\left\{\begin{array}{ccc}1 & \text { if } & H_{r / g} \leq T h_{r} \cap H_{b / g} \leq T h_{b} \\ 0 & \text { else }\end{array}\right.$

The thresholds are selected in such a way to isolate natural variations of hue from hue variations caused by lens shading. 


\section{Stage III: Clustering of Hue-flat regions}

The blocks that are flat in terms of hue variation are then clustered together based on their spatial distribution, and average hue values. The Figure 3 illustrates these how a grid of blocks classified as hue-flat (1) and non-hue-flat (0) are clustered together.

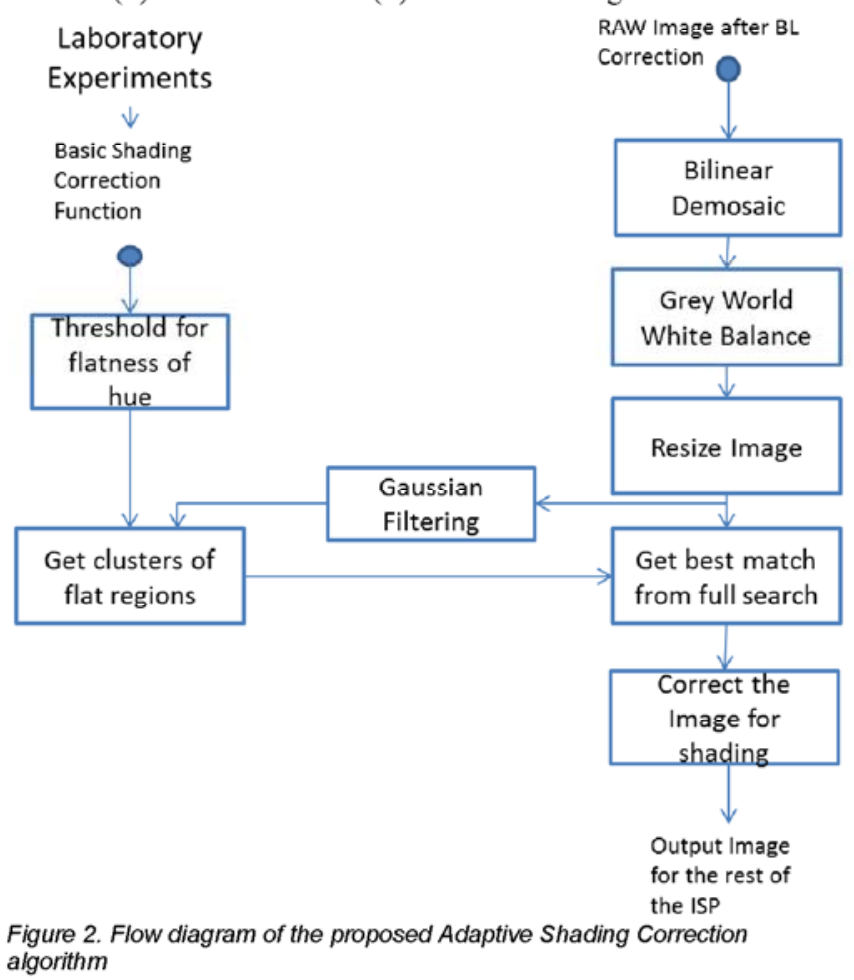

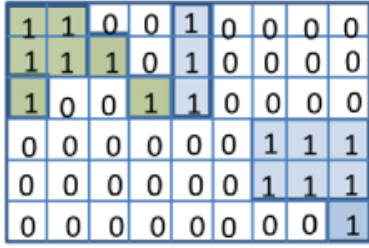

Hue-flat blocks

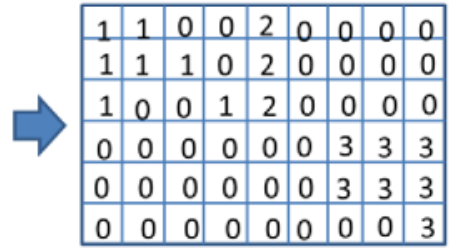

Clusters
Figure 3: Example of the clustering operation

\section{Stage IV: Finding the best shading correction function}

Once the hue-flat regions are identified according to stage III, next step is to find the best shading correction mesh. To find the best shading correction mesh, the average shading mesh is gradually modified in steps along three dimensions for individual color channels.

The modification of the average shading mesh is done on 3dimensions: shifting in the horizontal $(\Delta \mathrm{x})$ and vertical $(\Delta \mathrm{y})$ directions and scaling (S). These 3 dimensions are briefly illustrated in Figure 4.

For each modification of the average shading mesh, the total hue variation of all clusters is calculated as in (3);

$$
E_{r / g}=\sum_{c}^{C} \sum_{i}(r / g-\bar{r} / g)^{2}
$$

where $\mathrm{C}$ is total number of clusters identified in stage III.

The best modification parameters for the red channel $\left(\Delta \mathrm{x}_{\text {best }, r}, \Delta \mathrm{y}_{\text {best }, r}\right.$ and $\left.\mathrm{S}_{\text {best },}\right)$, for the given image is found by the modification that yields in the lowest $\mathrm{E}_{r / g}$, as given in (4); $\left(\Delta x_{\text {best }, r}, \Delta y_{\text {best }, r}, S_{\text {best }, r}\right)=\underset{\Delta x, \Delta y, s}{\arg \min }\left(E_{r / g}\right)$

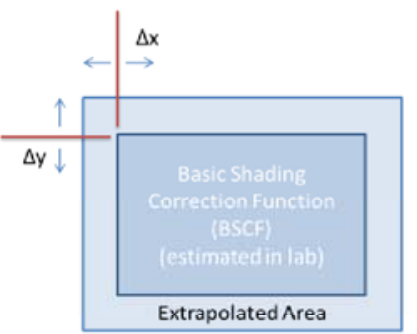

(a) Shifting in $\mathrm{x}$ and $\mathrm{y}$ direction

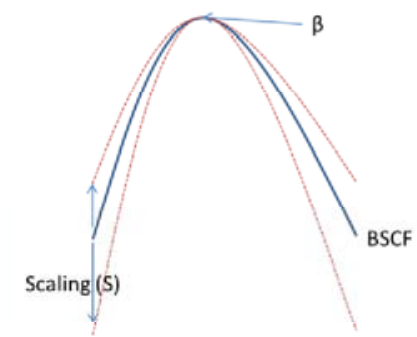

(b) Cross section of the ASM
Figure 4: Illustration of the Adaptation Dimensions

\section{Results and Discussion}

In this section the results obtained by the proposed method are presented and compared against the state-of-the-art techniques. The performance of the proposed Adaptive Shading Correction (ASC) method is compared against the Dynamic Mesh Shading Correction algorithm (DMSC). The shading correction experiments are carried out on 160 raw images captured with several camera units manufactured by OmniVision (Part No: OV8835). While traditional image quality metrics (such as SSIM or VQM) cannot be used for this purpose, as none of them take color shading artifacts in to consideration, we use the variance of hue in hue-flat regions as color shading metrics and average pixel saturation as the saturation measure.

The results in the Table I, indicate that the proposed ASC method out performs DMSC in terms of color shading measured as huevariance metrics, without compromising overall saturation.

Table I: Comparison of performance in terms of color shading and saturation

\begin{tabular}{|l|l|l|}
\hline Methic & $\begin{array}{l}\text { Hue-variance } \\
\text { in hue-flat } \\
\text { blocks }\end{array}$ & $\begin{array}{l}\text { Average pixel } \\
\text { Saturation }\end{array}$ \\
\hline $\begin{array}{l}\text { Dynamic } \\
\text { Mesh Shading } \\
\text { correction }\end{array}$ & 0.072 & 0.2165 \\
\hline $\begin{array}{l}\text { Adaptive } \\
\text { shading } \\
\text { correction }\end{array}$ & 0.064 & 0.2115 \\
\hline
\end{tabular}

Most of the published works on vignetting correction focus mainly on luminance shading correction. This results in significant color shading on the resultant image. More importantly, none of the methods specifically handle per-unit variation of vignetting. The proposed algorithm is unique in its adaptation technique, which handles per-unit variation of vignetting function without losing naturally occurring shadows in the image nor losing the overall saturation. Furthermore, the proposed algorithm does not rely on illuminant (light source) estimation from the image, compared to the current industry standard vignetting correction from DXO Labs [3]. 
(2)2016 Society for Imaging Science and Technology

DOI: 10.2352/ISSN.2470-1173.2016.18.DPMI-249
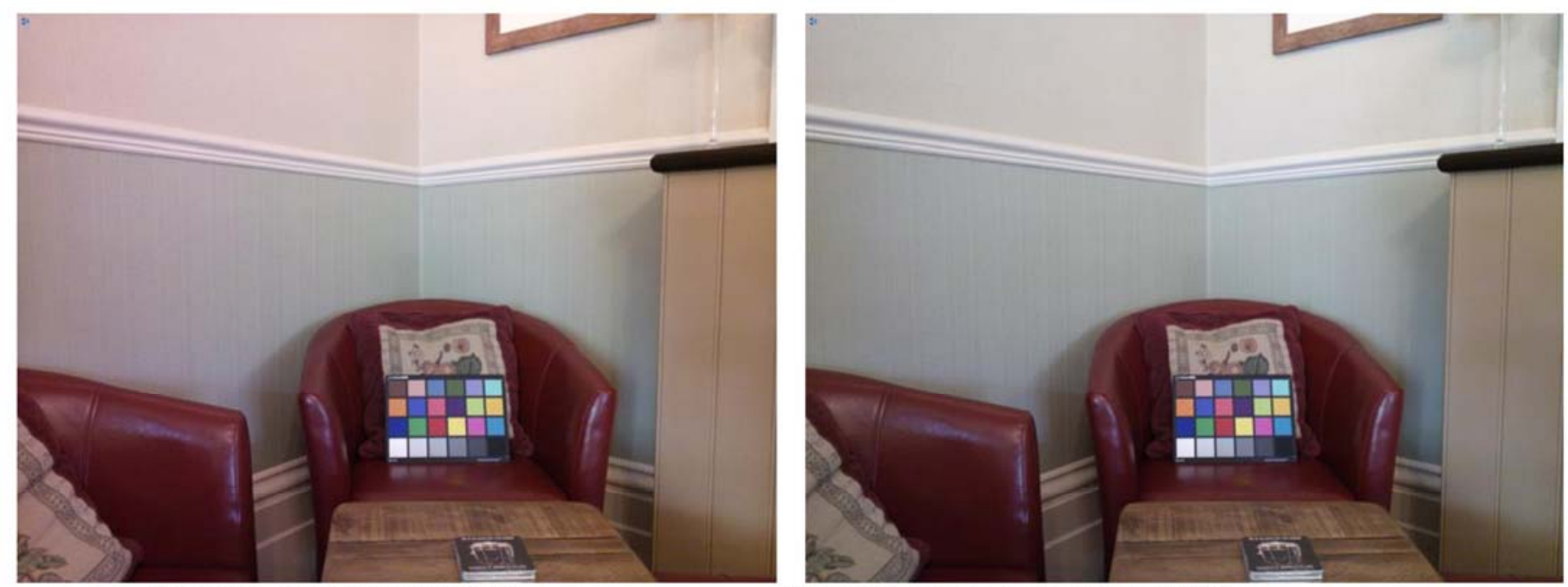

Vignetting corrected with DMSC

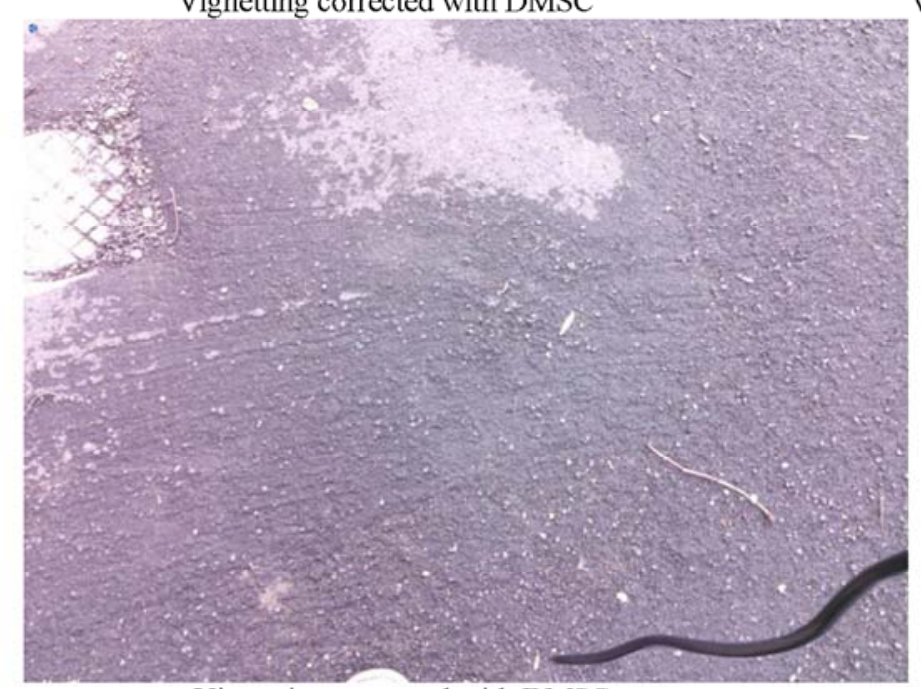

Vignetting corrected with proposed ASC

Vignetting corrected with DMSC

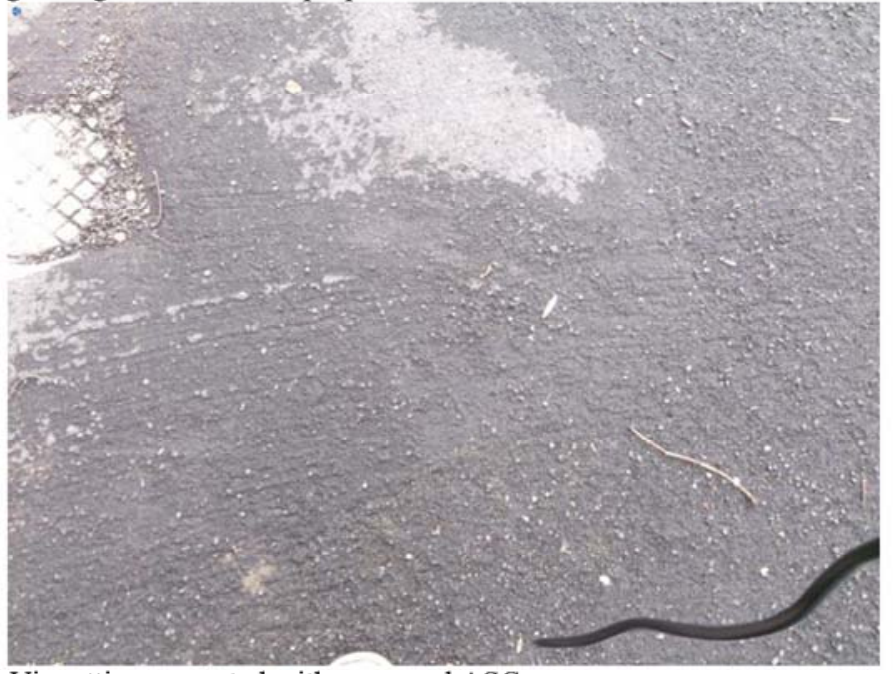

Vignetting corrected with proposed ASC

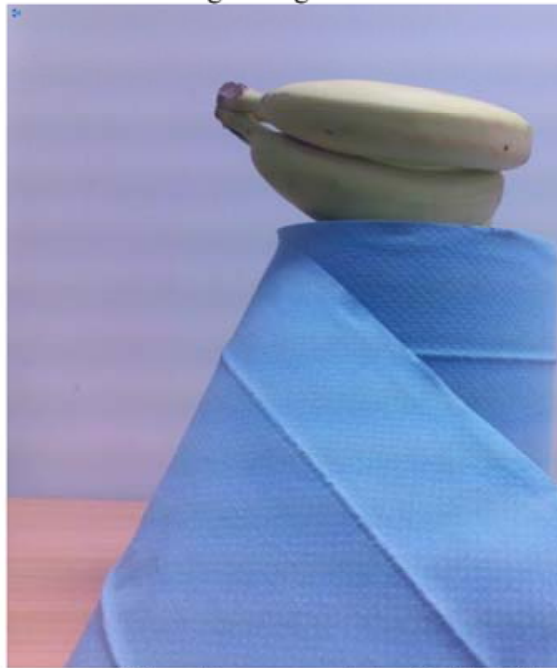

Vignetting corrected with DMSC

Vignetting corrected with proposed ASC

Figure 7: Comparison of Visual results for three images 


\section{Conclusions}

This paper presents a novel method to correct color shading artifacts in images. The adaptive method utilizes three dimensions of adaptation to find an optimal shading correction function for a given image by analyzing the hue variation in the image. The proposed method does not rely on light source identification and account for the variations in color shading brought about by both sensor-to-sensor variation and light source variation. The visual and objective results indicate that the proposed method significantly outperforms its competition.

\section{References}

[1] W. Yu, Y. Chung, J. Soh,; "Vignetting Distortion Correction Method for High Quality Digital Imaging", in proceedings of $17^{\text {th }}$ International Conference on Pattern Recognition, 2004

[2] Y. Zheng, S. Lin, S. Kang,; "Single-Image Vignetting Correction", IEEE Transactions on Pattern Analysis and Machine Intelligence, vol.31, no.12, pp.2243-2256, Dec. 2009

[3] US Patent 2009/0251567 Al, Processing method for relative illumination phenomenon on a digital image and associated processing system.

[4] T. Tajbakhsh, "Color lens shade compensation achieved by linear regression of piece-wise bilinear spline functions," Proc. of SPIE $7537,75370 \mathrm{P}, 2010$
[5] I. Dinstein, F. Merkle, L. Tinwai; W. Kwan,; "Imaging system response linearization and shading correction," in Proceedings. 1984 IEEE International Conference on Robotics and Automation. pp.204209,1984

\section{Author Biography}

Varuna De Silva received PhD in Electronic Engineering from University of Surrey, UK in 2011. From 2011 to 2013 he worked at University of Surrey as a post-doctoral research fellow. Since 2013 he has been with Apical Ltd, as a senior researcher in Imaging systems.

Viaceslav Chesnokov received his B.Sc and M.Sc degrees in physics and applied mathematics from the Moscow Institute of Physics and Technology, Russia, in 1991, and PhD in Computer Science (Computational Neuroscience) from De Montfort University, UK, in 2001. He is a cofounder and CTO of Apical Limited, a UK company specializing in advanced imaging technologies. His current research interests include Image Processing, Neural Networks and Artificial Intelligence.

Daniel Larkin received his PhD in Electronic Engineering from Dublin City University, Ireland in 2008. From 2007 to 2010 he worked at Analog Devices in Ireland as a digital hardware design Engineer. Since 2010 he has held a variety of R\&D roles within Apical Imaging. His current position is the Head of Hardware Engineering. 